\title{
A PESQUISA BRASILEIRA EM ESTUDOS MÉTRICOS DA INFORMAÇÃO: proximidade entre pesquisadores de destaque e áreas afins
}

\author{
Maria Cláudia Cabrini Grácio* \\ Ely Francina Tannuri de Oliveira*
}

RESUMO:

Palavras-chave: Estudos Métricos da Informação no Brasil. Análise de Citação e Cocitação de Autores. Interdisciplinaridade em Estudos Métricos.

\footnotetext{
* Doutora em Lógica pela Universidade Estadual de Campinas, Brasil. Professora do Departamento de Ciencia da Informação da Universidade Estadual Julio de Mesquita Filho, Brasil. Docente permanente do Programa de Pós-Graduação em Ciência da Informação Universidade Estadual Julio de Mesquita Filho, Brasil.

E-mail: cabrini@marilia.unesp.br.

** Doutora em Educação pela Universidade Estadual Paulista Júlio de Mesquita Filho, Brasil. Livre-Docente em Estudos Bibliométricos pela Universidade Estadual Paulista Júlio de Mesquita Filho, Brasil. Docente permanente do Programa de Pós-Graduação em Ciência da Informação da Universidade Estadual Paulista Júlio de Mesquita Filho, Brasil. E-mail: etannuri@gmail.com.
}

\section{INTRODUÇÃO}

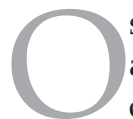

$s$ Estudos Métricos da Informação têm apresentado um aumento acentuado na ciência mainstream, desde o início dos anos de 1980, quando se consolidam como uma disciplina científica, principalmente em função do rápido desenvolvimento das tecnologias, da disponibilidade de grandes bases de dados bibliográficas e softwares específicos (GLÄNZEL, 2003).

Fundamentados em recursos quantitativos como método de análise, os "Estudos Métricos da Informação" constituem o conjunto de conhecimentos relacionados à avaliação da 
informação produzida e são alicerçados na sociologia da ciência, na ciência da informação, matemática, estatística e computação. Referemse aos estudos de natureza teórico-conceitual quando contribuem para o avanço do conhecimento da própria temática, propondo novos conceitos e indicadores, bem como reflexões e análises relativas à área. São de natureza metodológica quando se propõem a dar sustentação aos meta-estudos nas diferentes áreas do conhecimento (OLIVEIRA; GRÁCIO, 2011). Entre suas subáreas, encontram-se: Bibliometria, Cientometria, Webometria, Patentometria, Altmetria e a Informetria, esta última com a maior amplitude e a primeira constituindo a origem dos Estudos Métricos.

No Brasil, alinhados com a tendência mundial, esses estudos se desenvolveram a partir da década de 1970 e apresentaram grande avanço nos anos de 1990. Meneghini e Packer (2010) apontam que o número de publicações brasileiras em Estudos Métricos da Informação cresceu 13 vezes no período de 1990 a 2006, bastante acima do crescimento da ciência brasileira no geral (5,6 vezes).

Nesse contexto, as pesquisas brasileiras em Estudos Métricos têm alcançado grande inserção nos periódicos internacionais, especialmente a partir de 2006, permanecendo com uma tendência ascendente até os dias atuais. Além do significativo crescimento da área, registra-se a existência de muitos investigadores brasileiros atuantes em distintas áreas de conhecimento, com pesquisas destinadas à análise do comportamento da ciência e para políticas científicas.

Considera-se que a pesquisa bibliométrica contemporânea é destinada a três grupos-alvo principais, que determinam seus tópicos e subáreas (GLÄNZEL, 2003), a saber:

- - $\begin{array}{rrr}\text { Bibliometria para profissionais } \\ \text { da Bibliometria (G1): pesquisas }\end{array}$ bibliométricas "de base", desenvolvidas pelos bibliometristas, nas quais os conceitos, indicadores e procedimentos bibliométricos são o objeto de estudo, na busca do desenvolvimento, reflexão e debate da Bibliometria, a fim de propiciar o próprio avanço conceitual-teóricometodológico.

- - Bibliometria aplicada às disciplinas científicas (G2): pesquisas bibliométricas que compõem os procedimentos metodológicos de meta-estudos destinados à análise e avaliação do comportamento científico das distintas áreas do conhecimento. Forma o maior e mais diversificado grupo de interesse na Bibliometria.

- - Bibliometria para Política Científica e Gestão (G3): pesquisas bibliométricas desenvolvidas com a finalidade de orientar políticas científicas. Glänzel (2003) considera este o grupo mais importante da Bibliometria contemporânea.

Considerando o exposto, esta pesquisa tem como objetivo identificar os pesquisadores brasileiros e internacionais de destaque nos Estudos Métricos da Informação, bem como a proximidade estabelecida entre eles, na perspectiva de pesquisadores brasileiros com atuação consolidada na área. Ainda, identificar as disciplinas, áreas ou subáreas do conhecimento, que contribuem para o desenvolvimento teórico, epistemológico e metodológico, de forma a oferecer subsídios e instrumentos para os EMI, na perspectiva desta comunidade. Os objetivos propostos são analisados à luz da natureza das pesquisas declaradas por essa comunidade científica (G1, G2 ou G3), a fim de contribuir para identificar e visualizar as possíveis similaridades e diferenças teórico-metodológicas adotadas por esses grupos.

Justifica-se a relevância desta pesquisa em função da carência de estudos cientométricos que analisem os pesquisadores brasileiros de destaque em uma área, a partir da avaliação dos próprios pares. Em geral, os estudos bibliométricos buscam identificar a elite de uma área científica a partir da observação dos seus resultados (produtos), por meio da análise da intensidade da sua produção científica e da contagem das citações na literatura da área, em uma determinada janela temporal. Considerase que também o olhar e a compreensão dos próprios pares possibilitam identificar os autores com contribuição relevante para o desenvolvimento da área, independentemente do período e do volume da produção científica. Desse modo, esta abordagem fundamenta-se em recursos qualitativos, ao passo que os estudos baseados na contagem da produção científica e suas citações se fundamentam em recursos 
quantitativos, ao analisar a literatura científica reconhecida na área em estudo, socializada sob a forma de artigos, capítulos de livro e livro, entre outros.

Desse modo, esta proposta metodológica, que retira os dados a partir da compreensão da própria comunidade científica, contribui para ampliar os procedimentos cientométricos que possibilitam visualizar o conjunto de pesquisadores consignado e reconhecido como aquele que sinaliza os novos caminhos do conhecimento de uma determinada área.

\section{ANÁLISE DE CITAÇÃo COCITAÇÃO}

As citações podem ser analisadas como reflexo de uma comunidade discursiva. Seu estudo baseia-se em análises das frequências das citações a autores ou documentos e das frequências de coocorrência (cocitação) entre as mesmas, possibilitando a visualização de uma área do conhecimento.

Uma citação é tomada como indicador objetivo e claro da comunicação científica. Ela permite a identificação de grupos de cientistas e suas publicações, com a finalidade de evidenciar os pesquisadores de maior impacto de uma área, apontando os paradigmas das comunidades formadas, procedimentos metodológicos pertinentes e os pesquisadores de maior impacto, a "vanguarda" que constrói o novo conhecimento na área (GLÄNZEL, 2003). Considera-se, assim, que a lista dos autores mais citados representa o conjunto de pesquisadores que definem a estrutura teórico-metodológica de uma área do conhecimento científico (SMIRAGLIA, 2011).

Os estudos de citação e seus indicadores se baseiam em dois tipos ${ }^{1}$ de análises: univariáveis e relacionais (ou de ligação). Estas últimas

\footnotetext{
Nos indicadores bibliométricos e cientométricos univariáveis, cada elemento em estudo é medido segundo uma dimensão escolhida. Callon, Courtial e Penan (1995) os denominam indicadores de atividade e consideram que estes seguem um modelo que concebe a ciência como uma atividade produtiva normal, medindo volume de produção e impacto, por meio do cômputo do número de publicações e de citações, por pesquisador, instituição, periódico, país, ano e área, entre outros. Para estes autores, os indicadores relacionais remetem a um modelo diferente de análise do desenvolvimento científico e tecnológico, centrado nas interações presentes na área, no qual se privilegia o estudo da dinâmica das múltiplas interlocuções, associações e relações. Entre os indicadores relacionais, destacam-se os estudos de colaboração científica, redes de coautoria e de citação, análises de cocitação, de acoplamento bibliográfico e de coocorrência de termos, entre outros.
}

permitem conhecer as relações estruturais de conectividade teórico-metodológica de uma área, a proximidade, a vizinhança, a associação e a interlocução estabelecida entre os documentos, pesquisadores, periódicos, entre outros, como reconhecidos pela comunidade científica. Para isso, consideram a observação simultânea de duas citações, ou seja, sustentam-se em medidas que observam a distância ou proximidade entre dois documentos, autores, periódicos, entre outros. Desse modo, as análises relacionais das citações são destinadas a mapear as proximidades temáticas, teóricas e/ou metodológicas entre artigos, autores, periódicos, países ou outras unidades de análise, destacando-se a Análise de Cocitação (GRÁCIO, 2016).

Nesse contexto, observa-se que a estrutura da ciência é gerada por padrões de correconhecimento. Quando os cientistas concordam em relação ao que é relevante para a construção do conhecimento em uma área, ao citarem o que é significante, definem as estruturas das áreas do conhecimento científico (SMALL, 2004).

Entre as análises relacionais de citação, destacam-se os estudos de cocitação, os quais buscam identificar, evidenciar e visualizar as proximidades e/ou semelhanças entre os pesquisadores, segundo medidas de "proximidade" destinadas a estimar as similaridades e as dissimilaridades entre os indivíduos analisados (ROSTAING, 1996). Envolvem, assim, a observação simultânea de dois elementos, como unidade de análise, isto é, baseiam-se sempre em pares de elementos para a observação da característica (relação) analisada.

Desse modo, quando autores ou documentos são cocitados, os autores citantes estão atribuindo correconhecimento, bem como criando uma associação de significados. A cocitação pode ser usada para estabelecer o núcleo científico em um determinado tema ou área (SMALL, 2004). Este núcleo pode se referir a artigos, pesquisadores, instituições ou países, entre outros.

A Análise de Cocitação de Autores (ACA) trata especificamente da frequência com que dois autores são citados de forma conjunta em uma área, indicando a proximidade teórica ou metodológica entre eles, na perspectiva da comunidade citante. 
A força da cocitação entre dois autores citados pode ser facilmente determinada a partir do número de vezes que os autores foram citados juntos e indica a intensidade das ligações relacionais (SMALL, 1973).

Segundo McCain (1990), o princípio dos estudos de cocitação é analisar a estrutura intelectual de uma determinada área, pressupondo-se que pesquisadores com problemas de pesquisa semelhantes citem fontes informacionais similares e próximas. Nesse sentido, a ACA pode ser definida como a análise de um conjunto de autores ou pesquisadores organizados estruturalmente em rede (social e cognitiva) em uma determinada área. É possível identificar, a partir da construção das redes de cocitação, as proximidades e clusters entre os autores mais citados e, portanto, com maior impacto. Ainda, aqueles que ocupam posições mais centrais na rede de conhecimento gerada, com maior proximidade com os demais, assim como aqueles com posições mais periféricas dentro da área estudada (WHITE; GRIFFITH, 1981).

Na visão clássica, a análise de citação e cocitação nascem a partir do estudo do conjunto de referências de um documento científico. Todavia, já em 1990, McCain indica que há distintas formas para a seleção do conjunto de autores para a Análise de Cocitação de Autores (ACA), além da clássica, por seleção dos autores nas listas de referências dos documentos. Cita outras fontes, tais como: conhecimento pessoal, consulta a pesquisadores da área de estudo, artigos de revisão (isolados ou em agregado), listas de participantes em eventos científicos da área e autores presentes em listas de premiação.

Ainda outros estudos indicam a ampliação da forma de seleção dos autores para a análise de citação e cocitação, entre eles Cottrill et al. (1989), que usam a amostragem por bola de neve como procedimento de levantamento para a identificação das obras altamente citadas. Também Penan (1989) utiliza, como procedimento de seleção dos autores que participarão da ACA, o conjunto daqueles que publicam em revistas da área em estudo.

Nesta pesquisa, os autores, e não os documentos, constituem a unidade de análise, por isso a cocitação indica a frequência com que dois autores são citados juntos em um artigo.

\section{PROCEDIMENTOS METODOLÓGICOS}

O conjunto dos pesquisadores brasileiros com atuação consolidada na área dos Estudos Métricos da Informação foi determinado a partir dos anais dos Encontros Brasileiros de Bibliometria e Cientometria (EBBCs), considerado o evento brasileiro de maior relevância e especificidade temática nesta área. O critério adotado para compor o universo dos pesquisadores exigiu formação mínima de doutor e, pelo menos, duas apresentações de trabalhos orais nas edições de 2010, 2012 e 2014. Dos 226 participantes com comunicações orais, 44 atenderam a esse critério, incluindo os autores desta pesquisa, que optaram por não responder ao questionário. Desta forma, o universo de pesquisa totalizou 42 pesquisadores.

Por meio do software SurveyMonkey, enviou-se um questionário, instrumento considerado fidedigno e científico especialmente em pesquisa social, para esse conjunto de 42 pesquisadores, com a apresentação dos objetivos da pesquisa e as questões relacionadas aos objetivos propostos: quais pesquisadores brasileiros e também os de âmbito internacional destacam-se na área de Estudos Métricos da Informação; quais disciplinas, áreas ou subáreas do conhecimento contribuem para o desenvolvimento epistemológico, teórico e metodológico dos EMI; e, finalmente, a qual(is) dos três grupos-alvo (G1, G2 e G3), estabelecidos por Glänzel (2003), estão voltadas as atividades de pesquisa do respondente.

Do universo de 42 pesquisadores brasileiros, $18 \quad(\sim 43 \%)$ responderam ao questionário enviado: 17 deles, no próprio software; e um, por e-mail, justificando sua não participação, apesar de compreender a relevância da pesquisa.

Dos 17 participantes, 15 responderam à questão relativa aos pesquisadores brasileiros de destaque na área dos EMI. Foram citados 35 autores brasileiros, dentre eles alguns fizeram parte do grupo dos 42 pesquisadores respondentes. É importante destacar que não ocorreram autocitações.

Do total de 35 autores brasileiros mencionados pelos participantes, por meio da lei do Elitismo de Price, destacaram-se os 9 autores mais citados, correspondendo a ter sido citado por pelo menos 6 respondentes. 
Do total de 67 autores estrangeiros mencionados pelos participantes, aplicando a lei do Elitismo também para este conjunto de autores, destacaram-se 8 autores estrangeiros, correspondendo a ter sido citado por pelo menos 5 respondentes.

Realizou-se a análise multivariada de clusters hierárquicos pelo método Ward, com medida de similaridade pela de distância euclidiana, para o conjunto total de 17 pesquisadores, sendo 9 brasileiros e 8 estrangeiros a fim de se agrupar os autores em função de suas similaridades conforme as citações feitas pelos respondentes e as áreas de atuação autodeclarada por estes. Para os dois grupos gerados por esta análise, foram calculadas as estatísticas descritivas - média e coeficiente de variação-, a fim de se comparar a tendência central e de dispersão entre eles.

Construiu-se, então, a matriz quadrada e simétrica $17 \times 17$, registrando-se as frequências de cocitação entre os 17 pesquisadores. A partir desta matriz, gerou-se a matriz de proximidade normalizada pelo Cosseno de Salton (CS). A seguir, a rede de cocitação foi gerada a partir da matriz valorada por CS e calculados os indicadores de densidade da rede e de centralidade de grau, de proximidade e de intermediação para os 17 pesquisadores, usando-se o software Ucinet.

Em relação às disciplinas, áreas ou subáreas do conhecimento, apontadas pelos respondentes como aquelas que trazem contribuição significativa para o desenvolvimento epistemológico, teórico e metodológico dos EMI, registraram-se 23 disciplinas e áreas distintas. Foram consideradas com maior contribuição para os EMI aquelas mencionadas por pelo menos 2 respondentes, em um total de 13 áreas, subáreas e disciplinas.

\section{ANÁLISE E DISCUSSÃO DOS RESULTADOS}

Em relação aos autores brasileiros de destaque nos Estudos Métricos da Informação, dos 17 autores participantes, 15 responderam a esta questão. Registraram-se 128 citações para 35 autores distintos, sendo 32 pesquisadores e 3 autores institucionais (grupo UNESP, grupo UFRGS e grupo UFSCar), resultando uma média de 8,5 citações por respondente e média de 3,7 citações por autor citado.
Em relação às três universidades destacadas pelos pares, salienta-se que todas possuem cursos de graduação em Biblioteconomia, Pós-Graduação em Ciência da Informação e grupos de pesquisa em Estudos Métricos. Entre as instituições mais produtivas na temática, presentes no artigo de Meneghini e Packer (2010), estão presentes a UFSCar e UFRGS. A UNESP aparece como a instituição cuja produção científica é a mais expressiva no Grupo de trabalho Produção e Comunicação da Informação em Ciência, Tecnologia \& Inovação (GT7) da ANCIB, no período de 2011 a 2015, na pesquisa de Vogel et al. (2016).

A Tabela 1 apresenta os 9 pesquisadores mais citados, com 75 (59\%) citações do total de 128 citações. Considera-se que os autores mais citados refletem de forma significativa o pensamento relativo aos pesquisadores brasileiros de destaque nos Estudos Métricos da Informação, uma vez que os respondentes compõem $36 \%$ dos pesquisadores participantes do universo de pesquisa, oriundos do fórum máximo brasileiro da temática em estudo (EBBC).

Tabela 1. Pesquisadores brasileiros mais citados, segundo grupo-alvo autodeclarado pelo respondente ${ }^{1}$

\begin{tabular}{|l|c|c|c|c|c|}
\hline $\begin{array}{l}\text { Pe s q u is a d or e s } \\
\text { mais citados }\end{array}$ & G1 & G2 & G3 & \# citações & $\%$ \\
\hline $\begin{array}{l}\text { Rogério Mugnaini } \\
\text { (USP) }\end{array}$ & 5 & 11 & 6 & 14 & 93 \\
\hline $\begin{array}{l}\text { Jacqueline Leta } \\
\text { (UFRJ) }\end{array}$ & 3 & 9 & 2 & 10 & 67 \\
\hline $\begin{array}{l}\text { Rogério Meneghi- } \\
\text { ni (SciELO/USP) }\end{array}$ & 3 & 8 & 5 & 10 & 67 \\
\hline $\begin{array}{l}\text { Ida R. Chitto Stu- } \\
\text { mpf (UFRGS) }\end{array}$ & 4 & 4 & 2 & 9 & 60 \\
\hline $\begin{array}{l}\text { Lea M.L.S.Velho } \\
\text { (UNICAMP) }\end{array}$ & 3 & 6 & 2 & 8 & 53 \\
\hline $\begin{array}{l}\text { Ely Tannuri de } \\
\text { Oliveira (UNESP) }\end{array}$ & 2 & 4 & 2 & 6 & 40 \\
\hline $\begin{array}{l}\text { Raimundo N. M. } \\
\text { Santos (UFPE) }\end{array}$ & 3 & 4 & 3 & 6 & 40 \\
\hline $\begin{array}{l}\text { Sonia Caregnato } \\
\text { (UFRGS) }\end{array}$ & 3 & 3 & 2 & 6 & 6 \\
\hline $\begin{array}{l}\text { Suzana Mueller } \\
\text { (UNB) }\end{array}$ & 3 & 3 & 2 & 6 & 6 \\
\hline
\end{tabular}

Fonte: Elaboração dos autores

${ }^{1}$ Cada respondente pode se autodeclarar como atuante em mais de um dos três grupos-alvo.

Participantes autodeclarados por grupo-alvo: 5 respondentes em G1; 11 em G2; e 8 em G3. 
Pelo menos 40\% dos respondentes reconhecem a contribuição dos 9 pesquisadores, presentes na Tabela 1, para o desenvolvimento das pesquisas em Estudos Métricos no Brasil, com destaque para R. Mugnaini, indicado por 93\% dos respondentes. Os pesquisadores J. Leta, R. Meneghini, I. R. C. Stumpf e L. Velho foram citados pela maioria deles.

R. Mugnaini foi citado como pesquisador de destaque por todos os respondentes que se autoidentificam como atuantes em G1, grupo dedicado ao desenvolvimento conceitual-teóricometodológico da área dos EMI, e I. Stumpf, por quatro destes respondentes.

Os respondentes que centram suas pesquisas na bibliometria aplicada (G2) também destacaram o pesquisador R. Mugnaini, ao ser citado por todos. Outros pesquisadores ressaltados por este grupo foram: J. Leta e R. Meneghini.

Entre os oito respondentes atuantes em bibliometria voltada para a política científica (G3), seis deles reconhecem a contribuição de R. Mugnaini para esse grupo-alvo, seguido de R. Meneghini, com cinco citações.

A maioria dos autores presentes na Tabela 1 é (ou foi) bolsista de produtividade em pesquisa do $\mathrm{CNPq}$, o que sugere a consistência das suas atuações científicas, bem como o reconhecimento das instituições formais de apoio à pesquisa em relação à consolidação dos Estudos Métricos da Informação, no Brasil. Esses pesquisadores são, em geral, líderes de grupos de pesquisa e credenciados em Programas de Pós-Graduação em Ciência da Informação em suas instituições de origem, evidenciando que se encontram predominantemente concentrados em espaços formais de produção do conhecimento.

Rogério Mugnaini é docente do Departamento de Biblioteconomia e Documentação da Escola de Comunicações e Artes (ECA) da Universidade de São Paulo (USP), tem graduação em Estatística e formação de pós-graduação (Mestrado e Doutorado) em Ciência da Informação, com estágios de pesquisa na Universidad Carlos III de Madrid e na Leiden University. Coordena projetos de pesquisa nacionais e internacionais e centra suas investigações nos seguintes temas: bibliometria, cientometria, avaliação de produção científica nacional, indicadores, fontes de informação e política científica. Sua formação e temáticas de atuação explicam o reconhecimento dos pesquisadores de todos os grupos-alvo da bibliometria contemporânea.

Rogério Meneghini graduou-se em Química e doutorou-se em Bioquímica pela Universidade de São Paulo. Dedica-se ao estudo de comunicação científica e da ciência brasileira, desde a mensuração com indicadores até a avaliação e suas possibilidades de aplicação em política científica, fixando nestas temáticas suas atividades. Foi cocriador do projeto SciELO de revistas.

Jacqueline Leta possui graduação em Ciências Biológicas e mestrado e doutorado em Gestão, Educação e Difusão em Ciências. Conduz pesquisas na área da Bibliometria/Cienciometria, com ênfase nas análises da produção científica brasileira, focando nas temáticas Ciência e Saúde e Ciência e Gênero. Suas publicações têm foco na ciência brasileira e comunicação cientifica. Em 2008, organizou o $1^{\circ}$ Encontro Brasileiro em Bibliometria e Cientometria e, em 2009, a Conferência Internacional em Cientometria e Informetria. Realizou o pós-doutorado sob a coordenação de W. Glänzel, um dos principais nomes da cientometria mundial.

Lea Velho é professora titular em Estudos Sociais da Ciência e da Tecnologia da Universidade Estadual de Campinas. Possui graduação e mestrado em Engenharia Agronômica, pela UNESP, e doutorado em Science and Technology Policy, pela University of Sussex, Reino Unido. Atua nos seguintes temas: dinâmica da produção e uso do conhecimento, cooperação internacional em C\&T, avaliação de políticas e atividades de C\&T, formação de recursos humanos para pesquisa, indicadores de C\&Te Política Científica e Tecnológica.

Ida C. Stumpf doutorou-se em Ciências da Comunicação pela Universidade de São Paulo e realizou estágio pós-doutoral sênior na Universidad Carlos III de Madrid. Entre 2007 e 2008, coordenou o GT7 - Produção e Comunicação de Informação em Ciência Tecnologia \& Inovação (CT\&I), da Associação Nacional de Pesquisa e Pós-Graduação em Ciência da Informação - ANCIB. Desenvolve pesquisa em temáticas voltadas à Comunicação Científica, Produção Científica, Bibliometria, Cientometria, Estudos de Usuários e Ciência da Informação.

Observa-se que os autores brasileiros destacados e reconhecidos pelos pares advêm 
de formação acadêmica tanto da própria área da Ciência da Informação quanto de distintas áreas do conhecimento, especialmente de Biologia/ Biomédicas, Saúde e Exatas, porém com atuação e produção científica focada nos Estudos Métricos da Informação. Essa característica também foi observada no estudo de Meneghini e Packer (2010). Embora a abordagem metodológica destes estudos se constitua numa disciplina consolidada por especialistas, pesquisadores das diferentes áreas, em especial da Biologia/Biomédicas, Saúde e Exatas, esses autores mencionados têm dado importantes contribuições para a criação de indicadores relevantes, assim como para as reflexões e aplicações destes em política acadêmica e científica.

Os autores presentes na Tabela 1, mencionados pelos pares nos questionários respondidos para esta pesquisa, também se destacam em outras pesquisas, que tratam da análise da produção e da citação presente na literatura científica da área dos Estudos Métricos da Informação.

Entre os autores brasileiros mais produtivos no periódico Scientometrics, Mattos e Job (2008) destacam os pesquisadores J. Leta e L.Velho. Entre os autores mais citados na produção científica do GT7 do ENANCIB, no período de 2003 a 2010, Oliveira e Grácio (2011) destacam: R. Mugnaini, J. Leta, R. Meneghini, I. Stumpf, L. Velho, R. N. M. dos Santos, S. Caregnato e S. Mueller.

Desse modo, os estudos bibliométricos que buscaram identificar a elite científica e a frente de pesquisa dos Estudos Métricos da Informação a partir da análise da sua produção científica, seja na ciência mainstream, seja no fórum de debate científico nacional - ENANCIB -, apresentam resultados similares aos obtidos nesta pesquisa, por meio do olhar dos pares.

A Tabela 2 apresenta os 8 autores estrangeiros de destaque nos Estudos Métricos da Informação, na compreensão dos 17 respondentes.

Dentre os oito pesquisadores estrangeiros, observam-se autores clássicos ou fundadores dos estudos métricos, tais como Price, Garfield, Pritchard e Bradford, além de pesquisadores contemporâneos, que vêm consolidando os estudos da área, como Glänzel, Leydesdorff, Rousseau e Spinak. Os autores basilares - como Price, Glänzel, Garfield e Leydesdorff - foram citados por pesquisadores com atuação em todos os grupos-alvo, sendo mencionados por pelo menos 50\% deles. Dentre os pesquisadores atuantes em G1, somente um deles não identificou Glänzel e Price como fundamento para os Estudos Métricos.

Tabela 2. Pesquisadores estrangeiros mais citados, segundo grupo-alvo do respondente ${ }^{1}$

\begin{tabular}{lllllc|c|}
\hline $\begin{array}{l}\text { Pesquisadores in- } \\
\text { ternacionais mais } \\
\text { citados }\end{array}$ & G2 & G3 & \# citações & $\%$ \\
\hline $\begin{array}{l}\text { D. de Solla Price } \\
\text { (E.U.A.) }\end{array}$ & 5 & 9 & 6 & 12 & 71 \\
$\begin{array}{l}\text { Wolfgang Glänzel } \\
\text { (Bélgica) }\end{array}$ & 5 & 9 & 5 & 12 & 71 \\
$\begin{array}{l}\text { Eugene Garfield } \\
\text { (E.U.A.) }\end{array}$ & 3 & 7 & 6 & 8 & 47 \\
$\begin{array}{l}\text { Loet Leydesdorff } \\
\text { (Holanda) }\end{array}$ & 3 & 6 & 4 & 7 & 41 \\
$\begin{array}{l}\text { Alan Pritchard (Rei- } \\
\text { no Unido) }\end{array}$ & 3 & 4 & 2 & 6 & 35 \\
$\begin{array}{l}\text { Samuel C. Bradford } \\
\text { (Reino Unido) }\end{array}$ & 1 & 4 & 2 & 5 & 29 \\
$\begin{array}{l}\text { Ronald Rousseau } \\
\text { (Bélgica) }\end{array}$ & 2 & 5 & 3 & 5 & 29 \\
$\begin{array}{l}\text { Ernesto Spinak (Uru- } \\
\text { guai) }\end{array}$ & 3 & 3 & 2 & 5 & \\
\hline
\end{tabular}

Fonte: Elaboração dos autores

${ }^{1}$ Cada respondente pode se autodeclarar como atuante em mais de um dos três grupos-alvo.

Participantes autodeclarados por grupo-alvo: 5 respondentes em G1; 11 em G2; e 8 em G3.

Pelo exposto, considera-se que o conjunto dos 17 pesquisadores participantes tem clareza e compreensão da ampla gama de estudiosos necessários para fundamentar as bases teóricas e/ou metodológicas dos Estudos Métricos da Informação, ao mencionarem autores da sociologia da ciência, da comunicação científica, de políticas científicas, da Bibliometria clássica até a Bibliometria contemporânea.

A Tabela 3 apresenta os pesquisadores brasileiros e estrangeiros mais citados agrupados em dois grupos (A e B) segundo suas similaridades em função das citações feitas pelos respondentes e as áreas de atuação autodeclaradas por estes e as estatísticas 
descritivas de cada um dos grupos, a saber: Média Aritmética e Coeficiente de Variação (CV).

O grupo A é formado por 8 pesquisadores, entre eles 5 brasileiros e 3 estrangeiros, e o grupo B por 9 pesquisadores, 4 brasileiros e 5 estrangeiros, o que mostra certa equidade entre brasileiros e estrangeiros nos dois grupos.
Observa-se que para todos os indicadores presentes na Tabela 3, os CVs do grupo B são menores que $30 \%$ e que os respectivos do grupo A. Ainda, também no grupo A, com exceção dos indicadores relativos ao G3, os indicadores apresentam variação (CV) menor que $30 \%$.

Tabela 3. Agrupamentos dos pesquisadores brasileiros e estrangeiros mais citados e respectivas estatísticas descritivas.

\begin{tabular}{|c|c|c|c|c|c|c|c|c|c|}
\hline & $\begin{array}{l}\text { Pesquisadores mais } \\
\text { citados }\end{array}$ & G1 & $\%$ & G2 & $\%$ & G3 & $\%$ & $\begin{array}{c}\# \\
\text { citações }\end{array}$ & $\%$ \\
\hline \multirow{8}{*}{ GRUPO A } & D. Price & 5 & $100 \%$ & 9 & $82 \%$ & 6 & $75 \%$ & 12 & 71 \\
\hline & G. Wolfgang & 5 & $100 \%$ & 9 & $82 \%$ & 5 & $63 \%$ & 12 & 71 \\
\hline & J. Leta & 3 & $60 \%$ & 9 & $82 \%$ & 2 & $25 \%$ & 10 & 67 \\
\hline & R. Meneghini & 3 & $60 \%$ & 8 & $73 \%$ & 5 & $63 \%$ & 10 & 67 \\
\hline & L.Velho & 3 & $60 \%$ & 6 & $55 \%$ & 2 & $25 \%$ & 8 & 53 \\
\hline & E. Garfield & 3 & $60 \%$ & 7 & $64 \%$ & 6 & $75 \%$ & 8 & 47 \\
\hline & I. Stumpf & 4 & $80 \%$ & 4 & $36 \%$ & 2 & $25 \%$ & 9 & 60 \\
\hline & R. Mugnaini & 5 & $100 \%$ & 11 & $100 \%$ & 6 & $75 \%$ & 14 & 93 \\
\hline \multirow{13}{*}{ GRUPO B } & S. Caregnato & 3 & $60 \%$ & 3 & $27 \%$ & 2 & $25 \%$ & 6 & 40 \\
\hline & S. Mueller & 3 & $60 \%$ & 3 & $27 \%$ & 2 & $25 \%$ & 6 & 40 \\
\hline & E. Oliveira & 2 & $40 \%$ & 4 & $36 \%$ & 2 & $25 \%$ & 6 & 40 \\
\hline & R. Santos & 3 & $60 \%$ & 4 & $36 \%$ & 3 & $38 \%$ & 6 & 40 \\
\hline & L.Leydesdorff & 3 & $60 \%$ & 6 & $55 \%$ & 4 & $50 \%$ & 7 & 41 \\
\hline & A.Pritchard & 3 & $60 \%$ & 4 & $36 \%$ & 2 & $25 \%$ & 6 & 35 \\
\hline & S.Bradford & 1 & $20 \%$ & 4 & $36 \%$ & 2 & $25 \%$ & 5 & 29 \\
\hline & R.Rousseau & 2 & $40 \%$ & 5 & $45 \%$ & 3 & $38 \%$ & 5 & 29 \\
\hline & E.Spinak & 3 & $60 \%$ & 3 & $27 \%$ & 2 & $25 \%$ & 5 & 29 \\
\hline & Média do Grupo A & 3,9 & $78 \%$ & 7,9 & $72 \%$ & 4,3 & $53 \%$ & 10,4 & $66,1 \%$ \\
\hline & Média do Grupo B & 2,6 & $51 \%$ & 4,0 & $36 \%$ & 2,4 & $31 \%$ & 5,8 & $35,9 \%$ \\
\hline & CV do Grupo A & $26 \%$ & $26 \%$ & $28 \%$ & $28 \%$ & $45 \%$ & $45 \%$ & $21 \%$ & $21 \%$ \\
\hline & CV do Grupo B & $28 \%$ & $28 \%$ & $25 \%$ & $25 \%$ & $30 \%$ & $30 \%$ & $12 \%$ & $15 \%$ \\
\hline
\end{tabular}

Fonte: Elaboração dos autores 
Destaca-se que a principal diferença entre os grupos A e B é o maior dos pesquisadores participantes do primeiro grupo (A) entre os respondentes que se autodeclaram atuantes em G2, i.e., realizam estudos métricos metateóricos para a análise do desempenho científico das diferentes áreas do conhecimento. Os pesquisadores do grupo $\mathrm{A}$, em média, receberam o dobro de indicações daqueles do grupo B.
Na Figura 1, apresentam-se as relações de cocitação entre os 17 pesquisadores brasileiros e estrangeiros. O tamanho dos círculos é proporcional à quantidade de citações recebidas, e a espessura das linhas é proporcional à intensidade de cocitação entre os pesquisadores. Os círculos em vermelho correspondem aos pesquisadores brasileiros.

Figura 1 - Rede de cocitação normalizada por CS entre os autores mais citados.

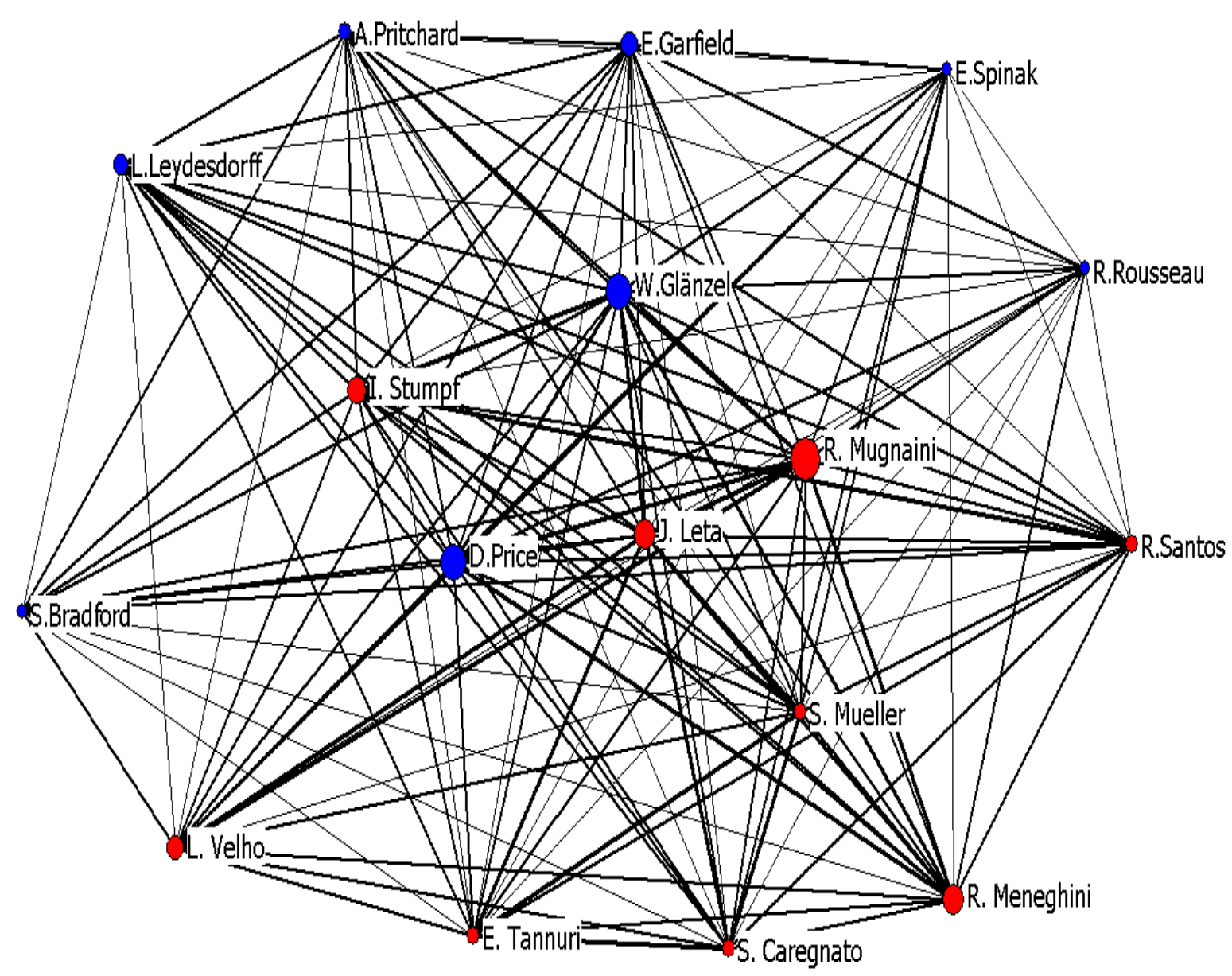

Fonte: Elaboração dos autores

A rede de cocitação é totalmente conectada com $98 \%$ de densidade, indicando um alto grau de coesão no grupo de pesquisadores. A relação mais intensa de cocitação ocorre entre duas pesquisadoras brasileiras - S. Mueller e S. Caregnato -, com valor igual a 1, significando que as autoras foram citadas sempre de forma conjunta. Ainda, destaca-se a alta intensidade de cocitação entre um pesquisador estrangeiro e uma brasileira 
- W. Glänzel e J. Leta -, igual a 0,91. Esta realizou o pós-doutorado sob a coordenação de W. Glänzel, um dos principais nomes da cientometria mundial, com decorrente intensa produção científica em coautoria. Com exceção de E. Spinak, S. Bradford e R. Rousseau, os demais pesquisadores foram cocitados com todos os outros pesquisadores da rede, todavia de forma menos intensa.

Em relação aos indicadores de centralidade de grau da rede, em ordem decrescente de intensidade, os autores mais centrais são: W. Glänzel, R. Mugnaini, J. Leta, D. Price, R. Meneghini e I. Stumpf. Dentre os mais centrais, destacam-se dois expoentes da bibliometria: um deles contemporâneo e o outro dos fundamentos da área. Em ordem crescente de intensidade, os autores menos centrais são: R. Rousseau, E. Spinak, S. Bradford e A.Pritchard.

Em termos de centralidade de proximidade e intermediação, exceto S.Bradford, R.Rousseau e E. Spinak, todos os outros pesquisadores apresentam os mesmos valores de índices para ambas as medidas de centralidade. S.Bradford, R. Rousseau e E. Spinak apresentam intermediação igual a zero e os menores valores de grau de proximidade.

A Tabela 4 apresenta as disciplinas, áreas ou subáreas do conhecimento consideradas, por pelo menos dois respondentes, como aquelas que trazem maior contribuição para o desenvolvimento epistemológico, teórico e metodológico dos Estudos Métricos da Informação, de forma a oferecer subsídios e instrumentos.

A partir da análise da Tabela 4, de acordo com as áreas do conhecimento do $\mathrm{CNPq}$, as grandes áreas de Exatas, Ciências Sociais Aplicadas e Ciências Humanas foram consideradas como as que contribuem significativamente para o desenvolvimento dos Estudos Métricos da Informação (EMI).

A grande área das Exatas, contemplada pela presença da Estatística, Ciência da Computação e Matemática, é citada pela maioria dos respondentes como de sustentação para o desenvolvimento dos EMI. Alguns respondentes especificam que a maior contribuição desta grande área refere-se aos aspectos metodológicos.
Tabela 4. Disciplinas, áreas ou subáreas do conhecimento, citadas por pelo menos 2 pesquisadores.

\begin{tabular}{|l|c|}
\hline Áreas, subáreas e disciplinas & Citações \\
\hline Estatística & 13 \\
\hline Sociologia da Ciência & 11 \\
\hline Ciência da computação & 9 \\
\hline Matemática & 9 \\
\hline Ciência da Informação & 7 \\
\hline Cientometria & 3 \\
\hline Comunicação Científica & 3 \\
\hline Filosofia da Ciência & 3 \\
\hline História da Ciência & 3 \\
\hline Metodologia Científica & 3 \\
\hline $\begin{array}{l}\text { Política em ciência e tecnolo- } \\
\text { gia }\end{array}$ & 3 \\
\hline Bibliometria & 2 \\
\hline Lógica & 2 \\
\hline
\end{tabular}

Fonte: Elaboração dos autores

A grande área das Ciências Humanas aparece citada com cinco áreas, subáreas e disciplinas, a saber: Sociologia da Ciência, Filosofia da Ciência, História da Ciência, Política em ciência e tecnologia e Lógica. Todavia, com exceção da Sociologia da Ciência, citada pela maioria dos respondentes, as demais foram citadas por um número menos significativo (dois ou três) de respondentes. Alguns enfatizaram que essa grande área oferece importante contribuição epistemológica e teórica para os estudos métricos.

Em ordem decrescente de citação, a grande área das Ciências Sociais Aplicadas é representada por: Ciência da Informação, Cientometria, Comunicação Científica, Metodologia Científica e Bibliometria, com as quatro últimas disciplinas pertencentes à subárea Ciência da Informação.

\section{CONCLUSÕES}

Em relação ao primeiro objetivo apresentado, conclui-se que, na compreensão da comunidade brasileira - aqui representada pelos pesquisadores doutores participantes 
dos Encontros Brasileiros de Bibliometria e Cientometria (EBBCs), que compuseram o universo desta pesquisa -, há um grupo de estudiosos já consolidado na temática em estudo, constituído por brasileiros atuantes em diversas universidades e centros de pesquisa estaduais e federais de diferentes regiões do país. Esses pesquisadores, em geral, são reconhecidos por agências de fomento brasileiras, tendo o $\mathrm{CNPq}$ uma atuação destacada na área em estudo, tanto pela formação de recursos humanos quanto pelo conhecimento científico gerado.

Atuam em grupos de pesquisa e, em geral, são credenciados em Programas de PósGraduação em Ciência da Informação em suas instituições de origem, evidenciando que se encontram predominantemente concentrados em espaços formais de produção do conhecimento.

A rede de cocitação indica um alto grau de coesão no grupo de pesquisadores brasileiros e estrangeiros na temática em estudo, com denotada presença destes últimos. A relação mais intensa ocorre entre duas pesquisadoras brasileiras atuantes em universidades federais brasileiras de regiões distintas, evidenciando que a elite científica, na visão dos pares, não está restrita a uma única região do país. Além disso, constata-se o reconhecimento da paridade da atuação brasileira e estrangeira visualizada pela intensa cocitação entre um pesquisador estrangeiro e uma brasileira.

Em relação às áreas, subáreas e disciplinas do conhecimento, que contribuem para o desenvolvimento epistemológico, teórico e metodológico, foram destacadas as grandes áreas de Exatas, Ciências Humanas e Ciências Sociais Aplicadas, com a primeira delas sendo citada pela maioria dos respondentes. Com cinco disciplinas distintas - Sociologia da Ciência, Filosofia da Ciência, História da Ciência, Política em ciência e tecnologia e Lógica -, a grande área das Ciências Humanas aparece como a segunda mais citada. A Ciência da Informação, Cientometria, Comunicação Científica, Metodologia Científica e Bibliometria compõem as subáreas citadas dentro da grande área das Ciências Sociais.

Aponte-se, a guisa de conclusão, que os resultados encontrados, oriundos da compreensão da comunidade científica brasileira em estudos métricos, entendidos como uma avaliação qualitativa da área, destacam outras pesquisas, que se preocupam em analisar a produção e a citação da área de Estudos Métricos da Informação, consideradas aqui de natureza quantitativa. Ressalta-se, desse modo, a paridade entre os indicadores oriundos de dados qualitativos e quantitativos e a complementação das duas análises.

Artigo recebido em 20/01/2017 e aceito para publicação em 19/06/2017

\section{THE BRAZILIAN RESEARCH IN INFORMATION METRIC STUDIES: proximity among prominent researchers and related areas}

ABSTRACT: This research aims to identify and analyze the prominent Brazilian and international researchers in Information Metric Studies in Brazil, the proximity established among them from the Brazilian researchers' perspectives, the disciplines, areas or sub-areas of knowledge that contribute for the theoretical and methodological development in order to provide subsidies and instruments for the Information Metric Studies. The objectives are analyzed in light of the nature of the researches declared by this scientific community, in order to contribute to identify and visualize the possible similarities and theoreticalmethodological differences adopted by these groups. As research procedure, it was used the questionnaire as a data collection instrument. Out of the universe of 42 Brazilian researchers, $18(\sim 43 \%)$ responded to the questionnaire. The co-citation network indicates a high degree of cohesion in the group of Brazilian and foreign researchers, with major presence of the latter. Regarding the areas, sub-areas and disciplines of knowledge that contribute to the epistemological, theoretical and methodological development, we highlight the areas of Exact Sciences, Humanities and Applied Social Sciences, with the first one being cited by most respondents. The Human Sciences area appeared as the second most cited. The Information Science, Scientometrics, Scientific Communication, Scientific Methodology and Bibliometry compose the subareas mentioned within the Social Sciences area. We conclude pointing to parity among the indicators derived from qualitative and quantitative data as well as the completion of the two analyzes.

Keywords: Information Metric Studies in Brazil. Citation and Co-citation analysis of Authors. Interdisciplinary in Metric Studies. 


\section{REFERÊNCIAS}

CALLON, M.; COURTIAL, J-P.; PENAN, H. Cienciometría: la medición de la actividad científica - de la bibliometría a la vigilância tecnológica. Asturias: Ediciones Trea, S.L., 1995.

COTTRILL, C. A., ROGERS, E. M., \& MILLS, T. Co-citation analysis of the scientific literature of innovation research traditions. Knowledge: Creation, Diffusion, Utilization, II, 181-208, 1989.

GLÄNZEL, W. Bibliometrics as a research field: a course on theory and application of bibliometric indicators. 2003. Bélgica. Disponível em: <http://citeseerx.ist. psu.edu/ viewdoc/download?doi=10.1.1.97.5311\&rep= rep1\&type $=$ pdf $>$

GRÁCIO, M. C. C. Acoplamento bibliográfico e análise de cocitação: revisão teórico-conceitual. Econtros Bibli, v.21, n.47, p. 82-99, set./dez. 2016. Disponível em: <https://periodicos. ufsc.br/index.php/eb/article/view/15182924.2016v21n47p82>. Acesso em: 15 set. 2016.

McCAIN, K. Mapping author intellectual space: a technical overview. Journal of the American Society for Information Science, v.41, n. 66, p.433-443, 1990.

MENEGHINI, R.; PACKER, A. L. The extent of multidisciplinary authorship of articles on scientometrics and bibliometrics in Brazil. Interciencia, v.3, n.57, p.510-514, 2010.

OLIVEIRA, E. F. T. de; GRÁCIO, M. C. C. Visibilidade dos pesquisadores do Gt7 da ANCIB: um estudo de cocitações. Tendências da Pesquisa Brasileira em Ciência da Informação, v. 4, p. 234-252, 2011.
PENAN, H. Pour une gestion bibliometrique de l'information scienttique et technique des entreprises: application en theorie microeconomique et financihre. 1989. Tese (Doutorado em Sciences de Gestion) - Universite des Sciences Sociales-Toulouse I, Toulouse, France,1989.

ROSTAING, H. La bibliométrie e ses techniques. Tolouse: Sciences de la Société, 1996.

SMALL, H. On the shoulders of Robert Merton: towards a normative theory of citation. Scientometrics, v. 60, n.1, p. 71-79, 2004.

SMALL, H. Co-citation in the scientific literature: a new measure of the Relationship between two documents. Journal of the American Society for Information Science,v. 24, n.4, p. 265-269, 1973.

SMIRAGLIA, R.P. ISKO 11's Diverse Bookshelf: an editorial. Knowledge Organization, v. 38, n.3, p. 179-186, 2011.

VOGEL, M. J. M. et al. Análise da representação dos PPGCIs nos GTs 1, 2 e 7 do ENANCIB a partir do BENANCIB. In: Encontro Brasileiro de Bibliometria e Cientometria, 5, São Paulo. Anais..., 2016.Disponível em: < http://www. ebbc.inf.br/ebbc5/index.php/ebbc5/trabalhos>. Acesso em: 30 de agosto de 2016.

WHITE, H.D.; GRIFFITH, B. C. Author cocitation: a literature measure of intellectual structure. Journal of the American Society for Information Science \&Technology, v. 32, n. 2., p. 163-171, 1981. 